\title{
Local Changes in the Excitability of the Cerebellar Cortex Produce Spatially Restricted Changes in Complex Spike Synchrony
}

\author{
Sarah P. Marshall and Eric J. Lang \\ Department of Physiology and Neuroscience, New York University School of Medicine, New York, New York 10016
}

\begin{abstract}
Complex spike (CS) synchrony patterns are modulated by the release of GABA within the inferior olive (IO). The GABAergic projection to most of the IO arises from the cerebellar nuclei, which are themselves subject to strong inhibitory control by Purkinje cells in the overlying cortex. Moreover, the connections between the IO and cerebellum are precisely aligned, raising the possibility that each cortical region controls its own CS synchrony distribution. This possibility was tested using multielectrode recordings of CSs and simple spikes (SSs) in crus $2 \mathrm{a}$ of anesthetized rats. Picrotoxin or muscimol was applied to the cerebellar cortex at the borders of the recording array. These drugs induced significant changes in CS synchrony and in CS and SS firing rates and changes in post-CS pauses and modulation of SS activity. The level of CS synchrony was correlated with SS firing rate in control, and application of picrotoxin increased both. In contrast, muscimol decreased CS synchrony. Furthermore, when picrotoxin was applied only at the lateral edge of the array, changes in CS synchrony occurred sequentially across the recording array, with cells located in the lateral half of the array having earlier and larger changes in CS synchrony than cells in the medial half. The results indicate that a double-inhibitory feedback circuit from Purkinje cells to the IO provides a mechanism by which SS activity may regulate CS synchrony. Thus, CS synchrony may be a physiologically controlled parameter of cerebellar activity, with the cerebellum and IO comprising a series of self-updating circuits.
\end{abstract}

\section{Introduction}

The Purkinje cell has the unique ability of generating different types of spikes depending on which excitatory afferent is driving it: simple spikes (SSs) in response to granule cell activity and complex spikes (CSs) following climbing fiber activation (Eccles et al., 1964). The significance of this ability, and how these different spike types interact, has been debated for decades (for review, see Simpson et al., 1996). The original name for the CS, "inactivation response," makes explicit an aspect of this interaction, namely, the brief pause in ongoing SS activity that occurs following each CS (Granit and Phillips, 1956; Bloedel and Roberts, 1971). Subsequent studies have similarly focused on how CSs affect SSs. For example, short-term (hundreds of milliseconds) changes in SS rate and rhythmicity following a CS have been demonstrated (McDevitt et al., 1982; Sato et al., 1992; Schwarz and Welsh, 2001). CS activity can also trigger long-term plastic changes in the responsiveness of Purkinje cells to parallel fiber activity (for review, see Ito, 1989, 2002). Finally, tonic SS firing rate is often inversely related to CS firing rate: lesions of the inferior olive (IO) increase SS activity, and stimulation of the IO decreases it (Colin et al., 1980; Rawson and Tilokskulchai, 1981;

Received May 27, 2009; revised 0ct. 1, 2009; accepted 0ct. 2, 2009

This work was funded by grants to E.J.L. from the National Institute on Alcohol Abuse and Alcoholism (AA016566) and the National Institute of Neurological Disorders and Stroke (NS37028).

Correspondence should be addressed to Dr. Eric J. Lang, Department of Physiology and Neuroscience, New York University School of Medicine, 550 First Avenue, New York, NY 10016. E-mail: eric.lang@nyumc.org.

D01:10.1523/JNEUROSCI.3498-09.2009

Copyright $\odot 2009$ Society for Neuroscience 0270-6474/09/2914352-11\$15.00/0
Montarolo et al., 1982; Demer et al., 1985; Savio and Tempia, 1985).

What has received virtually no attention is the converse possibility, that SS activity could influence CS activity. There is a clear anatomical basis for such influence. Purkinje cell axons, the only cerebellar cortical efferents, mostly target the cerebellar nuclei. A significant percentage of cerebellar nuclear projection cells, perhaps as many as half, provide GABAergic input to the IO (Nelson and Mugnaini, 1989). Thus, a disynaptic feedback pathway exists from the cerebellar cortex to the IO, whose activity evokes CSs (Eccles et al., 1966a). Moreover, the second leg of this pathway, the nucleo-IO projection, provides a major input to the IO glomeruli (de Zeeuw et al., 1989a), in which reside the majority of electrical coupling sites between IO neurons (Sotelo et al., 1974; King, 1976; de Zeeuw et al., 1990). These intraglomerular GABAergic terminals are thought to play a major role in controlling the efficacy of coupling between IO neurons by regulating the conductance of the spine membrane, thereby helping determine the patterns of CS synchrony (Llinas et al., 1974; Sotelo et al., 1974; Lang et al., 1996).

The inhibitory nature of both links in the cerebellar cortico-IO pathway suggests that changes in SS activity likely cause correlated changes in CS synchrony levels. For example, because Purkinje cells are inhibitory (Ito et al., 1964, 1970), increased SS activity should decrease cerebellar nuclear activity, and thereby decrease GABA release in the IO, which in turn should cause an increase in CS synchrony (Lang et al., 1996; Lang, 2002). However, the rebound spikes and plateau potentials displayed by cerebellar nuclear neurons (Jahnsen, 1986a,b; Llinás 
and Mühlethaler, 1988) may mean that the translation of changes in cerebellar cortical output to action on the IO may not be so straightforward. Moreover, non-GABAergic cerebellar nuclear cells provide an excitatory input to several midbrain nuclei (including the parvocellular red nucleus and nucleus of Darkschewitsch) that in turn provide a largely, but perhaps not entirely (Weiss et al., 1990; Horn et al., 1998), excitatory input to the IO, including its glomeruli (de Zeeuw et al., 1989b; De Zeeuw and Ruigrok, 1994).

In this paper we report the results of manipulating the excitatory level of the cerebellar cortex by the local application of GABA-A antagonists and agonists. We show that the level of cortical output is correlated with CS synchrony levels, and provide evidence that the circuits between the IO and cerebellum, which anatomical studies have suggested form closed loops, do indeed function as such.

\section{Materials and Methods}

Experiments were performed in accordance with the National Institutes of Health guidelines for the care and use of laboratory animals. The experimental protocols were approved by the IACUC committee of New York University, School of Medicine.

Surgical and electrode implantation procedures. Adult female Sprague Dawley rats (225-300 g) were anesthetized with an initial intraperitoneal injection of ketamine $(100 \mathrm{mg} / \mathrm{kg})$ and xylazine $(8 \mathrm{mg} / \mathrm{kg})$. Starting $1-2 \mathrm{~h}$ after the initial injection, supplemental anesthesia (ketamine, $\sim 260 \mu \mathrm{g} /$ $\mathrm{kg} / \mathrm{min}$; xylazine, $\sim 4 \mu \mathrm{g} / \mathrm{kg} / \mathrm{min}$ ) was delivered continuously through a femoral vein catheter to maintain a constant anesthetic depth. A tracheotomy was performed to allow insertion of a breathing tube to permit mechanical ventilation of the animal and delivery of supplemental oxygen. Temperature was monitored rectally and maintained at $36-37^{\circ} \mathrm{C}$ by use of an electric heating pad. A paralytic, gallamine triethiodide (Flaxedil), was given just before the recording session to prevent movement-related artifacts.

The experiments required obtaining multielectrode recordings from crus 2a Purkinje cells during chemical stimulation of the surface of crus 2a. To access this lobule, the animal was placed in a stereotaxic apparatus and the skin, muscle, and bone overlying the cerebellum were resected. A platform was positioned on the surface of crus $2 \mathrm{a}$ and cemented into place. The platform, which served to stabilize the microelectrodes and protect the surface of the brain, was composed of an electron microscope grid attached to the surface of a thin sheet of silicon rubber and fixed to tungsten metal rods with epoxy. Glass microelectrodes filled with a 1:1 solution of $2 \mathrm{M} \mathrm{NaCl}$ and glycerol ( $\sim 2 \mu \mathrm{m}$ tip diameter) were individually inserted through the grid using a joystick-controlled 3-axis micromanipulator (Burleigh Instruments). A single electrode was attached to the micromanipulator with a wax droplet and pushed through the rubber platform into the cerebellar cortex. CS activity was isolated in the molecular layer, at a depth of 75-100 $\mu \mathrm{m}$ below the cortical surface, at which SS activity is absent. Once CS activity had been isolated, the wax was melted to release the electrode and the manipulator was attached to another electrode. Electrodes were spaced $\sim 250 \mu \mathrm{m}$ apart such that they formed a rectangular array of up to 4 mediolaterally running rows and 10 rostrocaudally directed columns.

In some experiments SS activity was recorded along with CS activity. In initial experiments, one or two electrodes of the array were inserted deeper (150-200 $\mu \mathrm{m}$ below the cortical surface) than was usual to record both SSs and CSs. However, it proved difficult to maintain SS recordings for the time needed to implant the electrode array and make recordings (4-6h) using electrodes that were only held in place by the silicon rubber platform. So, in most cases, the electrode array for recording CSs was implanted first, and then SS activity was isolated using an electrode that remained attached to the micromanipulator to allow positional adjustments to maintain good isolation.

Application of picrotoxin and muscimol to the cerebellar cortex. Solutions of picrotoxin $(2 \mathrm{mg} / \mathrm{ml})$ or muscimol $(300 \mu \mathrm{M})$ were made by dissolving the drugs in Ringers solution (in mm: $126 \mathrm{NaCl}, 26 \mathrm{NaHCO}_{3}$,
$3 \mathrm{KCl}, 1.2 \mathrm{KH}_{2} \mathrm{PO}_{4}, 1.6 \mathrm{MgSO}_{4}, 2 \mathrm{CaCl}_{2}, 5$ HEPES, and 15 dextrose). Picrotoxin was delivered either electrophoretically via a pipette using a cycle comprising $5 \mathrm{~s}$ pulse $(5 \mu \mathrm{A})$ and $10 \mathrm{~s}$ interpulse periods for the duration of the recording condition, or by means of a piece of Gelfoam (Pharmacia, Upjohn) soaked in the picrotoxin-Ringers solution in separate experiments. In most experiments, the pipette tip or the picrotoxinsoaked pieces of Gelfoam were positioned $\leq 1 \mathrm{~mm}$ from the lateral edge of the recording array. Pipettes were inserted $\sim 100 \mu \mathrm{m}$ below the cortical surface, whereas the Gelfoam piece was placed on the cortical surface. Muscimol was applied using the Gelfoam method only. In two control experiments, picrotoxin was applied rostral to the recording array using the Gelfoam method.

Intra-IO injection of lidocaine. In some experiments, intra-IO injection of $4 \%$ lidocaine (Abbott Labs) was used to demonstrate that the recorded activity was truly CSs evoked by olivocerebellar activity. To position the injection pipette correctly within the IO, a recording electrode was first inserted into the brainstem, and activity characteristic of the IO was located. Identification of the correct intra-IO location was based on observation of characteristic IO activity that was correlated with CS activity recorded from crus $2 \mathrm{a}$. The stereotaxic coordinates of this location were noted, the microelectrode withdrawn, and an injection pipette inserted to the same coordinates. The lidocaine injection was then made using a pressure injection system in which the pipette was connected, via a short length of polyethylene tubing, to a syringe. The tubing and syringe were both filled with the lidocaine solution. A microdialysis pump (CMA Microdialysis) was used to drive the injection at a steady rate of $0.1 \mu \mathrm{l} / \mathrm{min}$.

At the conclusion of the recordings, the lidocaine solution was replaced, while keeping the pipette in place, by one containing alcian blue dye, and a small injection was made to mark the injection site. Coronal sections of the brainstem were made on a freezing microtome and counterstained with cresyl violet to confirm the injection site.

Data acquisition. The recording system (MultiChannel Systems) consisted of 128 main amplifiers and corresponding preamplifier stages (total gain $=1000,0.2-8.0 \mathrm{kHz}$ bandpass filters), and a dual processor personal computer. The sample rate was set to $25 \mathrm{kHz} /$ channel. For each channel, a single-level voltage threshold was set to detect spikes. Both the spike times and waveforms were recorded. The activity from individual amplifier channels was monitored on a digital oscilloscope and on a virtual oscilloscope built using the MCRack software (MultiChannel Systems). In addition, a matrix panel for monitoring the spike activity from all channels simultaneously was generated using the MCRack software. In this panel, the spatial location of each electrode was mapped to a specific box in the matrix corresponding to its relative location on the brain surface. When a spike recorded by a particular electrode crossed the voltage threshold for detection, the box on the panel that corresponded to the electrode's spatial location on crus 2a flashed red. Observation of the panel thus allowed continuous monitoring of the level of activity in all electrodes. Any unusual activity detected in the matrix panel was investigated directly using the oscilloscope. In addition, the effects of the drug injections could be followed online by changes in the patterns of activity in the matrix panel. Note that in most channels only CSs were recorded, so that the panel could be used for continuous monitoring of CS activity. In cases in which both CS and SS activity were recorded on the same electrode, off-line spike sorting routines were used to separate the CSs and SSs.

Data analysis. Data analysis was performed with custom written programs in Igor Pro (Wavemetrics). Unless otherwise specified, all values are given as mean $\pm \mathrm{SD}$. Comparisons of means were made using Student's $t$ test.

In each experiment, a control period of $20 \mathrm{~min}$ or longer of spontaneous CS activity was recorded before application of picrotoxin or muscimol to the cerebellar cortex. The effects of a drug were typically observed within 20-30 min after the start of its application. Recordings taken after the onset of these effects were compared with those taken in control conditions to determine the effects of cortical excitation on CS synchrony.

Correlation coefficients were used to measure CS synchrony. These coefficients were calculated using standard methods (Gerstein and 
A

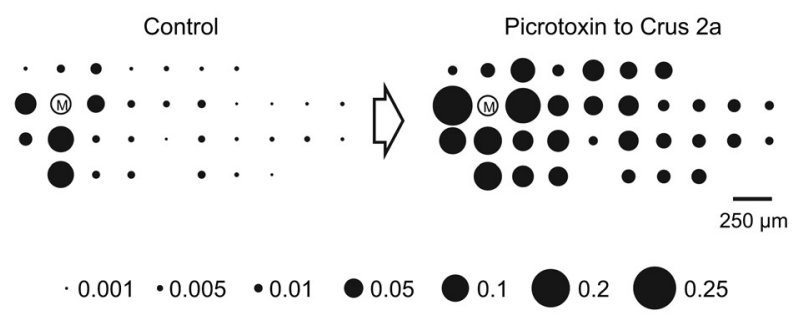

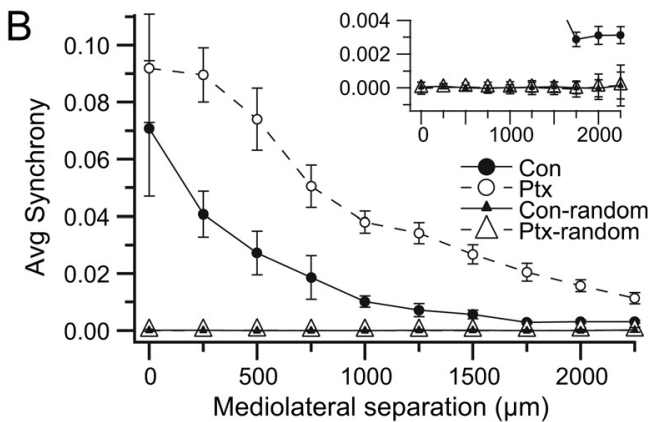

Figure 1. Application of picrotoxin to the cerebellar cortex increases the amplitude and spatial extent of CS synchrony. $\boldsymbol{A}$, Bubble plots show the effect of picrotoxin on CS synchrony with respect to one reference cell labeled $M$. Relative positions of the other electrodes in the recording array are indicated by circles. The area of each circle corresponds to the degree of synchrony between the activity of the cell at that location and the activity of cell $\mathrm{M}$. Interelectrode spacing was $250 \mu \mathrm{m}$ in this and all other experiments. Scale below bubble arrays gives $C(0)$ values for different-sized circles. $\boldsymbol{B}$, Graph of average synchrony from all cell pairs $(n=528)$ in the array as a function of the mediolateral separation between cells in a pair, in control (Con, filled circles) and after picrotoxin ejection from pipette (Ptx, unfilled circles). Synchrony curves with triangles are the averages of 50 randomized sets of spike trains made using the control (small filled triangles) and picrotoxin (large unfilled triangles) datasets of the experiment shown in the rest of this figure. Inset shows lower part of main graph with an expanded ordinate scale. Error bars are SEM, except for the control and picrotoxin random data curves, which are $2 \cdot$ SD to show $\sim 95 \%$ confidence limits.

Kiang, 1960; Sasaki et al., 1989). The spike train of a cell was represented by the function $X_{(i)}$, where $i$ represents the time step $(i=1,2, \ldots, n)$, and $X_{(i)}=1$ if the onset of a CS occurred in the ith time step and otherwise $X_{(i)}=0$. Each time step was equal to $1 \mathrm{~ms}$. $Y_{(i)}$ was defined the same as $X_{(i)}$, but represented the spike train of a second cell. The crosscorrelation coefficient, $C(t)$, was then calculated using the following formula: $C(t)=\left[\Sigma\left(X_{(i)}-X_{\text {avg }}\right) \cdot\left(Y_{(i-t)}-Y_{\text {avg }}\right)\right] / \sqrt{ }\left[\Sigma\left(X_{(i)}-X_{\text {avg }}\right)^{2}\right] \cdot$ $\left[\Sigma\left(Y_{(i)}-Y_{\text {avg }}\right)^{2}\right]$, where $X_{\text {avg }}=\Sigma X_{(i)} / n$ and $Y_{\text {avg }}=\Sigma Y_{(i)} / n$.

The summations run from $1, \ldots, n$, and $t$ represents the time lag between compared times of the spike trains.

The degree of synchronous CS activity was defined as the zero-time cross-correlation coefficient, $C(0)$, using the equation for $C(t)$ with $t=0$. A time bin of $1 \mathrm{~ms}$ was used for the analysis; thus, in order for two CSs to be considered synchronous, their onsets had to occur in the same $1 \mathrm{~ms}$ time step.

\section{Results \\ Application of picrotoxin to the cerebellar cortical surface increases CS synchrony levels and widens its spatial distribution}

Recordings of CS activity were obtained from 375 Purkinje cells located on the apex of the left lobule crus $2 \mathrm{a}$ of anesthetized adult female rats ( $n=17$ animals, $11-38$ cells/animal). In each experiment, an initial control period of spontaneous CS activity was recorded. Following this control period, picrotoxin was applied to the cerebellar cortex either by placing a small piece of Gelfoam saturated with the drug on the surface $(n=11)$ or by electrophoresis from a pipette that was inserted into the cortex to a depth of $\sim 100 \mu \mathrm{m}(n=6)$. By visual observation of the oscilloscope and matrix displays, the effects of picrotoxin on CS activity were usually apparent within 20-30 min (although shorter delays did occur). Thus, records from periods starting 20 min or more following initiation of picrotoxin application to the cortex, when a maximal or near maximal effect of drug appeared to have been realized, were compared with control records to determine the effects of cerebellar cortical excitation on CS activity.

In every experiment $(n=17)$, picrotoxin application to the cerebellar cortex caused an increase in CS synchrony. Figure 1 shows a representative experiment, in which surface picrotoxin application enhanced synchrony levels among cell pairs separated by all mediolateral distances measured, i.e., synchronous firing occurred more often, and between a wider range of cell pairs, than in control. In this experiment, 33 Purkinje cells were simultaneously recorded. Their relative positions in the record- ing array are indicated by the circles and the circled " $M$ " in Figure $1 A$ (up is rostral, left is lateral). The area of each circle is proportional to the level of synchrony between the CSs of the cell at that location and the CSs of the reference cell (cell M). As has been previously reported (Sasaki et al., 1989; Sugihara et al., 1993; Lang et al., 1999), the distribution of synchrony in control is such that the highest levels occur among cells located near the reference cell, with the level decreasing rapidly as cells are located farther from the reference cell along the longitudinal axis of the folium (Fig. $1 \mathrm{~A}$, horizontal direction) but remaining relatively constant with increasing separation along the transverse folial direction (Fig. $1 A$, vertical direction). This distribution is often referred to as the "rostrocaudal banding pattern of synchrony," as, in the rat, the transverse and longitudinal axes of most lobules are approximately aligned with the rostrocaudal and mediolateral axes of the brain, respectively. That this banding pattern, in which relatively high levels of synchrony occur mostly among cells located within $250-500 \mu \mathrm{m}$ of each other along the longitudinal axis of the lobule, reflects the predominant synchrony pattern is shown by the control curve in the plot of Figure $1 B$ (filled circles). This curve was generated by calculating the synchrony values of all cell pairs in the array and then obtaining averages for each set of cell pairs that were separated by the same distance in the mediolateral direction. As is typically the case, this curve is highest at the 0 and $250 \mu \mathrm{m}$ distances, reflecting the greater synchronization of CS activity among cells that are in the same or neighboring columns of the array.

After picrotoxin was applied to crus 2a, the level of synchrony between cell $\mathrm{M}$ and the other cells rose to much higher levels than in control, and the drop off in synchrony with mediolateral distance from cell $\mathrm{M}$ was more gradual (Fig. $1 \mathrm{~A}$, array to right of arrow). The picrotoxin condition curve (Ptx; unfilled circles) in Figure $1 B$ shows that this increase in synchrony distribution occurred throughout the array and thus is not specific to the particular choice for cell M. Similar increases in CS synchrony were observed in each of the experiments $(n=17$ experiments; $n=$ 3999 cell pairs), regardless of the method of drug delivery (Fig. $2 A, B)$. In sum, the results indicate that picrotoxin applied locally to the cerebellar cortex leads to increased CS synchrony.

Because the application of picrotoxin also causes an increase in CS firing rate (see three sections below), there will be more synchronous events between cells just by chance in this condition 
A

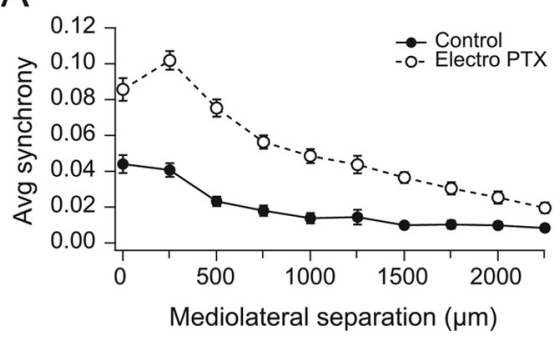

gles) were negligible, and far smaller than the differences between the curves for the true data (Fig. $1 B$, filled versus unfilled circles). Moreover, the typical shape of the synchrony curve is absent for the randomized data, as the curves based on randomized datasets are essentially flat in both conditions. Thus, changes in firing rates cannot explain the increase in synchrony observed here.

Figure 2. Summary of picrotoxin-induced increases in CS synchrony. $\boldsymbol{A}, \boldsymbol{B}$, Average synchrony levels according to application method: data from electrophoresis experiments are shown in $\boldsymbol{A}$ ( $n=6$ animals, 1346 pairs); data from Gelfoam experiments are shown in $\boldsymbol{B}$ ( $n=11$ animals, 2653 pairs). Both methods elicited significant increases in CS synchrony, although changes were slightly greater in the electrophoresis experiments.
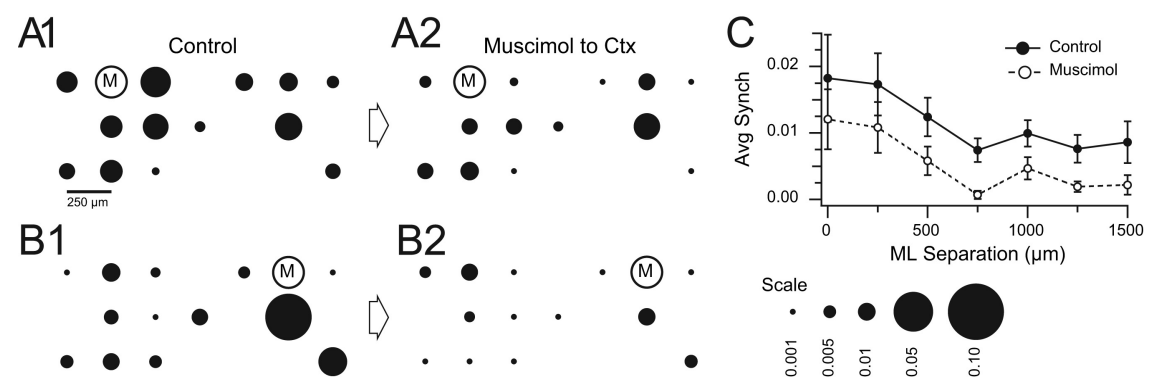

Figure 3. Muscimol applied to cerebellar cortex using Gelfoam lowers $C S$ synchrony. $A, B$, Bubble plots showing distribution of synchrony with respect to two reference cells in control $(\boldsymbol{A} 1, \boldsymbol{B} 1)$ and $40 \mathrm{~min}$ following the start of application of muscimol to the cortical surface adjacent to the recording array $(\boldsymbol{A 2}, \mathbf{B 2})$. The reduction in circle size in the muscimol condition compared with the control indicates that synchrony levels were reduced by muscimol. C, Plot of average synchrony of all cell pairs in the experiment as a function of the mediolateral separation between the cells in a pair. The muscimol curve (dashed line, unfilled circles) is lower than the control curve (solid line, filled circles). This difference was statistically significant for five of the seven separation distances. Error bars are SEM.

relative to control. Theoretically, the effect of changes in firing rate on synchrony is compensated for in the formula for calculating $C(0)$. In particular, in each term in the numerator, $X_{\text {avg }}$ is subtracted from $X_{(i)}$ in the multiplicand and $Y_{\text {avg }}$, is subtracted from $Y_{(i)}$ in the multiplier, where $X_{\text {avg }}$ and $Y_{\text {avg }}$ are the average firing rates of the cells. Therefore, when firing rates increase, the value of the terms representing synchronous events, $\left(1-X_{\text {avg }}\right)$. $\left(1-Y_{\text {avg }}\right)$, are smaller. The changes in average firing rates also affect the values of the other terms in the equation, but given the firing rates of CSs (even after picrotoxin), the synchrony terms are the dominant influence on the overall value of $C(t)$. Thus, changes in firing rates themselves should produce little to no change in the synchrony levels.

In the experiment shown in Figure 1 the average firing rate more than doubled with application of picrotoxin (control, $0.92 \pm 0.50 \mathrm{~Hz}$; picrotoxin, $2.00 \pm 0.83 \mathrm{~Hz} ; n=33 ; p=3.9 \times$ $10^{-7}$ ), which was typical (see below). Thus, to verify that there is no effect of firing rate increases on CS synchrony, the interspike intervals of each cell's spike train from the experiment were shuffled to make a set of randomized spike trains. Fifty such randomized datasets were made for each the control and picrotoxin conditions. The average synchrony distribution as a function of mediolateral separation was then calculated for each randomized dataset, and the fifty curves were averaged for each condition. The average synchrony curves were near zero at all mediolateral separations for the randomized data in both conditions (Fig. $1 B$, filled and unfilled triangles). Furthermore, the differences between the randomized synchrony curves from the control and picrotoxin conditions (Fig. $1 B$ inset, filled versus unfilled trian-

\section{Muscimol application to cerebellar cortex reduces CS synchrony}

To test whether decreasing cortical output reduced CS synchrony, two multiple electrode experiments were performed in which CS activity was recorded before and during Gelfoam application of muscimol to the surface of crus $2 a$. In both experiments, muscimol caused a significant reduction in CS synchrony (to $50 \%$ and $47 \%$ of control, $p=0.0033$ and $p=$ 0.0018). Results from one of these experiments are shown in Figure 3. In $A$ and $B$, the distribution of CS synchrony with respect to two reference cells is shown. After application of muscimol $(A 2, B 2)$, the synchrony levels dropped significantly from control levels $(A 1, B 1)$. In $C$, the average synchrony level for all cell pairs is plotted against mediolateral separation distance. A reduction in CS synchrony occurred for all separations, and was statistically significant $(p<0.05)$ in most cases (five of seven distances). In sum, these results show that increased GABA-A receptor-mediated activity in the cerebellar cortex leads to decreased CS synchrony.

\section{Picrotoxin-induced synchrony increases are spatially restricted}

Tract tracing studies have suggested that the pathways connecting the IO, cerebellar cortex, and cerebellar nuclei have a precise topography, and that they are all in register with each other (Groenewegen and Voogd, 1977; Groenewegen et al., 1979; Voogd and Bigaré, 1980; Van der Want et al., 1989; BuisseretDelmas and Angaut, 1993). Thus, together the pathways between these three brain regions form a series of closed loops, which suggests that each region of cortex should be able to influence the IO region from which it receives climbing fibers and thereby play a role in controlling its own CS synchrony patterns.

To test whether this is the case, time periods before full expression of picrotoxin's effects were analyzed (10-40 min after the start of the drug application) for experiments in which picrotoxin was applied to the cortex just lateral to the electrode array. Because picrotoxin was applied lateral to the array, the concentration of picrotoxin should rise first and fastest in the cortex underlying the lateral half of the recording array, as it is closest to the drug source (Fig. 4A). Therefore, if the anatomical connections between the cortex, cerebellar nuclei, and IO indeed function as a series of closed loops, CS synchrony should rise more rapidly among cells in the lateral half of the array, than among cells in the medial half of the array. To test this idea, the recording array was divided into near (unfilled circles) and far (filled cir- 

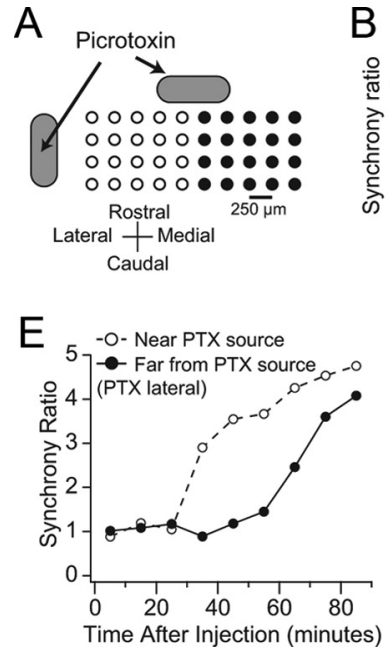
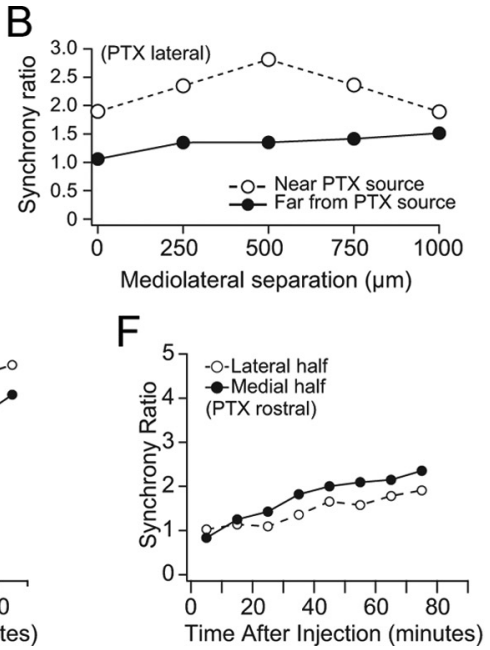
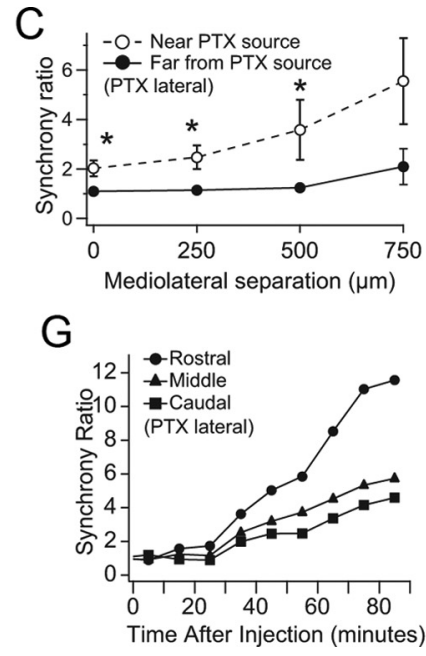
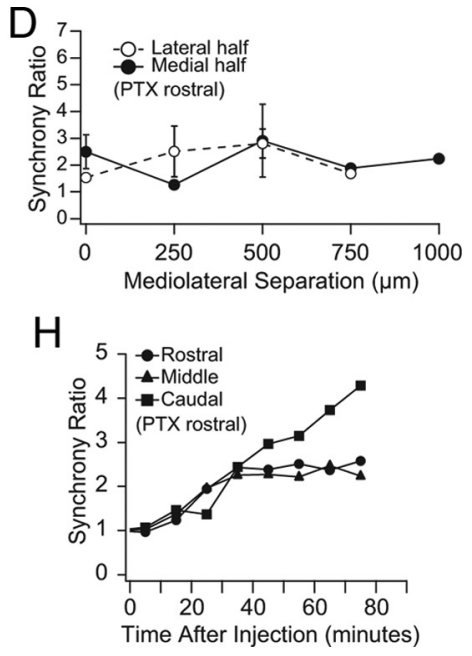

Figure 4. Picrotoxin-induced increases in (S synchrony are spatially restricted. $\boldsymbol{A}$, Schematic showing separation of an ideal recording array into near (unfilled circles) and far (filled circles) halves with respect to the standard picrotoxin injection site at the lateral edge of the recording platform. Both the standard application site (lateral to the array) and a rostral site used in some control experiments are indicated. In any one experiment only one of the sites was used. $\boldsymbol{B}$, Application of picrotoxin to lateral edge of array raises synchrony on the lateral half of the array first. Ratio of synchrony levels during the times shortly after the start of picrotoxin application to that of control as a function of mediolateral separation between cells for an experiment in which CS activity was recorded from 38 cells and picrotoxin was ejected from a pipette. The electrode array was divided into halves, and the cell pairs from the near and far halves were analyzed separately. CS synchrony more than doubled among PCs located on the side of the recording array near the picrotoxin site (unfilled circles) before large changes were observed on the far side of the array (filled circles). C, Average ratio of picrotoxin to control synchrony levels for all experiments in which picrotoxin was applied lateral to the array: $n=9$ experiments; picrotoxin applied using either Gelfoam ( $n=$ 7) or an injection pipette $(n=2)$; 94 "near" cells, 106 "far" cells. For each experiment, the near and far synchrony ratio curves were generated as described for $\boldsymbol{B}$. The curves shown in $\boldsymbol{C}$ are the averages of these nine curves. D, Same as $C$ for two experiments in which picrotoxin was applied rostral to the recording array. Note that there are no error bars for the points at 750 and $1000 \mu m$ because such separations occurred in only one experiment. Error bars in $\boldsymbol{C}$ and $\boldsymbol{D}$ are SEM, and asterisks in $\boldsymbol{C}$ indicate statistically significant differences ( $p$ values for 1 -sided $t$ test for distances of $0-750 \mu \mathrm{m}$ were $0.011,0.012,0.045$, and 0.055 ). There were no significant differences for data in $\boldsymbol{D}$. $\boldsymbol{E}$, Plot of synchrony ratios for near (lateral) and far (medial) halves of array for sequential 10 min periods following the start of picrotoxin application lateral to the array $(t=0 \mathrm{~min})$ in one experiment. The abscissa of each data point is the midpoint time of the 10 min period. Each ratio was calculated using the average $C S$ synchrony value for all cell pairs in the array half and dividing it by the average synchrony value in control for those cell pairs. $\boldsymbol{F}$, Same as $\boldsymbol{E}$ for an experiment in which picrotoxin was applied rostral to the array using Gelfoam. $\boldsymbol{G}, \boldsymbol{H}$, Same experiments and analysis as shown in $\boldsymbol{E}$ and $\boldsymbol{F}$, respectively, except that here the array was divided into three mediolaterally running rows of cells, rather than medial and lateral halves. The average synchrony of each row is plotted as a function of time from start of picrotoxin application.

cles) halves relative to the injection site, and CS synchrony was calculated for each half of the array separately, both during the control period and for periods starting 10-40 min after the initiation of the picrotoxin injection.

Direct comparison of the synchrony levels of the two halves of the array cannot be used to determine the relative effect of picrotoxin application, because the control (i.e., starting) levels of synchrony of the two halves are, in general, not the same. Instead, to control for those differences, the ratio of the synchrony during picrotoxin application to that in control was used as a measure of the relative effect of picrotoxin. This synchrony ratio was plotted as a function of cell separation along the longitudinal folial axis for each half of the array.

Figure $4 B$ shows the results of this analysis for a representative experiment in which CSs were recorded from 38 Purkinje cells simultaneously. The synchrony ratio curves in Figure $4 B$ show that synchrony among the cells in the near half of the array increased two- to threefold from control values, whereas a much more modest increase, or even no increase (i.e., ratio of 1 at $0 \mu \mathrm{m}$ separation), was observed among cells of the far half of the array.

Similar results were obtained in nine experiments $(n=201$ cells, 2194 cell pairs). The synchrony ratio curves from all nine experiments were averaged, and these average curves are shown in Figure $4 C$. The curves show that following the start of picrotoxin application to the cortex, CS synchrony among cells in the near half of the array typically rose two- to fivefold before there was virtually any change in synchrony among cells in the far half of the array. In comparison, for more delayed time periods (i.e., those used to generate Fig. 2) the ratios for both near and far halves were greater than one in all experiments, and there was a trend toward equalization of the ratios, though in most cases the synchrony ratio for the near half of the array remained higher than that for the far half.

These results suggest that cerebellar cortical influences on CS synchrony are spatially restricted to areas that are $<1 \mathrm{~mm}$ in width along the longitudinal folial axis (the half array width). It should be noted that in ML plots, such as in Figure $4 C$, which are created from all cell pairs in one half of the array, the cell pairs contributing to each data point are located at different distances from the lateral edge of the array and thus are at different distances from the Gelfoam, and therefore likely experience different concentrations of picrotoxin. To avoid this confound, one can plot the average synchrony among cells located in the same rostrocaudal column for each such column in the array. When this is done, in general, the synchrony ratios of the columns rise sequentially, with the ratios of laterally located columns rising before, or simultaneously with, more medial columns (data not shown); however, in this analysis, each data point is based on only one to three cell pairs, and for columns with only one cell, no value can be assigned for that column. Because of these limitations we did not pursue this analysis further; however, it is consistent with the above conclusion that CS synchrony may be controlled by regions whose widths are $<1 \mathrm{~mm}$, and suggests that these regions may be as small as $250 \mu \mathrm{m}$ wide in the longitudinal folial direction.

To test further that the localized changes in CS synchrony observed with lateral placement of the Gelfoam reflected the diffusion path of picrotoxin and the precise alignment of the cortico-nucleo-olivo-cortical circuits, rather than other factors, picrotoxin was applied by placing the Gelfoam piece at the rostral 
A1

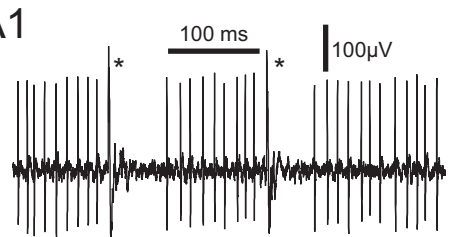

A2

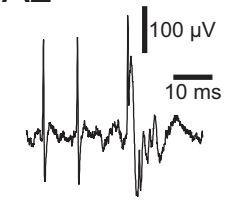

$\mathrm{C} 1$

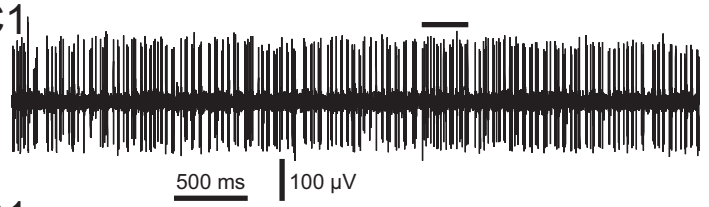

D1

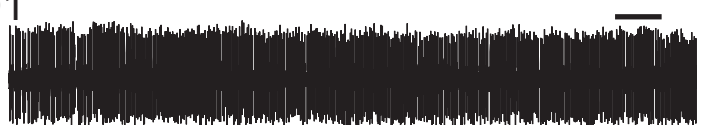

$\mathrm{B}$
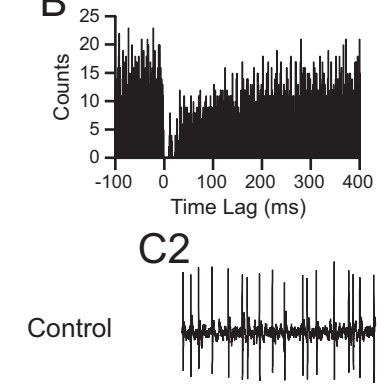
50 ms $\mid 100 \mu \mathrm{V}$

Picrotoxin to cortex
D2

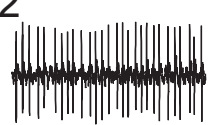

Figure 5. Increased SS firing following Gelfoam application of picrotoxin to cerebellar cortex. A, Demonstration of pause in SS activity following CSs. CSs are indicated by asterisks in $A 1$. The second CS in $A 1$, along with the two preceding SSs, is shown at an expanded time scale in $\boldsymbol{A} \mathbf{2}$. $\boldsymbol{B}$, CS-triggered histogram of $S \mathrm{~S}$ activity to demonstrate pause of SSs following a CS. CSs occurred at a time lag of $0 \mathrm{~ms}$. Note the near-complete suppression of $\mathrm{SS}$ activity for $20 \mathrm{~ms}$ following the $\mathrm{CS}$, which is followed by a period of reduced SS activity for several hundred milliseconds. Histogram compiled using 264 CSs as references. C, Extracellular recording of Purkinje cell activity in control. D, Activity of same cell after application of picrotoxin to surface of cortex adjacent to the recording electrode. Portions of recordings under bars in $\mathbf{C} \mathbf{1}$ and $\mathbf{D} \mathbf{1}$ are shown with an expanded time scale in $\mathbf{C}$ and $\mathbf{D} \mathbf{2}$, respectively.

the long delay between near and far halves of the array that was observed in $E$, and is consistent with rostrocaudal banding organization of CS synchrony.

\section{Cortical application of picrotoxin and muscimol alters spontaneous CS firing rates}

Increased SS activity following picrotoxin application (we demonstrate that this occurs later) should decrease cerebellar nuclear activity; thus, applying picrotoxin to the cerebellar cortex, in addition to increasing CS synchrony, should increase the average CS firing rate, given that blocking or eliminating GABAergic activity in the IO leads to increased spontaneous CS activity (Lang et al., 1996; Lang, 2002). Conversely, application of muscimol should decrease CS activity. Indeed, CS firing rates from periods $>30 \mathrm{~min}$ after the start of picrotoxin application were significantly higher than control rates (control, $0.82 \pm 0.62 \mathrm{~Hz}$; picrotoxin, $2.0 \pm 1.49 \mathrm{~Hz} ; n=375$ cells; $p<1 \times$

edge of the recording array in two experiments ( $n=32$ cells). The Gelfoam was centered as best as possible with respect to the mediolateral extent of the array, in an attempt to have picrotoxin spread symmetrically to the medial and lateral halves of the recording array. In one experiment the synchrony increase was greater on the medial half of the array, and the reverse was true in the other experiment; however, in both experiments the rise of CS synchrony on the two sides of the array were much more similar than was the case with picrotoxin applied laterally, both with regard to response amplitude and time course. The average ML synchrony ratio curves for these experiments are shown in Figure $4 D$. The application of picrotoxin rostral to the array produced a 1.5to 3-fold increase in CS synchrony. The amplitude of this increase is similar to that observed for the near (lateral) side of the array when picrotoxin was applied laterally (Fig. 4C). The important difference, however, is that in this case both sides of the array experienced a similar increase.

The time courses of the changes in CS synchrony caused by rostral and lateral application of picrotoxin also differed. To show this difference, the average value of CS synchrony for each half of the array was calculated for control and then for each 10 min period during application of picrotoxin. The ratios of the picrotoxin values to the control value are plotted in Figure $4 E$ and $4 \mathrm{~F}$ for experiments in which picrotoxin was applied lateral and rostral to the array, respectively. In the former case, the two curves diverge after $25 \mathrm{~min}$ because the ratio for the lateral half of the array rises, while the ratio for the medial array half remains near one for an additional 40 min before rising significantly. In contrast, in the latter case, in which picrotoxin was applied rostral to the array, the synchrony ratios for the lateral and medial halves of the array rise together in time (Fig. $4 F$ ).

The cells in an array can also be grouped according to which mediolaterally running row they are located. The synchrony changes were analyzed for three such rows (rostral, middle, and caudal) for each of the experiments shown in Figure 4, $E$ (results shown in $G$ ) and $F$ (results shown in $H$ ). In both experiments the synchrony ratios for all three rows begin to rise at the same time (35 min in $G$ and $15 \mathrm{~min}$ in $H$ ). This simultaneity is in contrast to $\left.10^{-16}\right)$. Conversely, muscimol decreased CS firing rates to $37 \%$ and $63 \%$ of control in two experiments $(p=0.0018$ and $p=$ 0.0024).

As was the case with synchrony, there appears to be local cortical control of CS firing rate. Comparison of firing rates of cells located in the near and far halves of the array from periods 10-20 min after the start of picrotoxin showed that although cells throughout the array had significantly increased CS firing rates (Near half: control, $0.63 \pm 0.41 \mathrm{~Hz}$; picrotoxin, $1.37 \pm 1.15 \mathrm{~Hz} ; n=95 ; p=1.4 \times 10^{-9}$; Far half: control, $0.80 \pm 0.55 \mathrm{~Hz}$; picrotoxin, $1.24 \pm 1.11 \mathrm{~Hz}, n=106 ; p=2.6 \times$ $\left.10^{-6}\right)$, the cells of the near half showed a greater relative increase $(117 \%$ versus $56 \%)$.

\section{Picrotoxin enhances spontaneous SS firing rates}

The simplest explanation of the effects of applying picrotoxin to the cerebellar cortex is that they are due to increased SS activity as a result of blocking GABAergic inhibition in the cerebellar cortex. To verify that this is the case, SS activity was recorded, along with CSs, before and after drug delivery. SSs were identified as such using several criteria, including depth of recording, combined recording of CS activity along with SSs on the same electrode, and demonstration of a pause in SS activity following a CS (Fig. $5 A, B)$. Application of picrotoxin to the cerebellar cortex typically produced a large increase in SS activity, as shown in Figure 5, C and $D$. Overall, SS firing rates increased from $26.1 \pm 16.8 \mathrm{~Hz}$ in control to $43.4 \pm 22.9 \mathrm{~Hz}$ after picrotoxin $(n=6$ animals, 7 cells; paired $t$ test, $p=0.02$ ).

\section{Changes in CS activity are correlated with SS firing rate changes}

The above results suggest that SS levels help determine the patterns of CS activity. Thus, we investigated the relationship between ongoing SS and CS activity directly by recording SSs (and CSs) from an electrode implanted into the middle of multielectrode arrays (in which CSs were recorded on the other electrodes). Control recording sessions were divided into sequential 5 min periods and the SS firing rate and average CS synchrony 

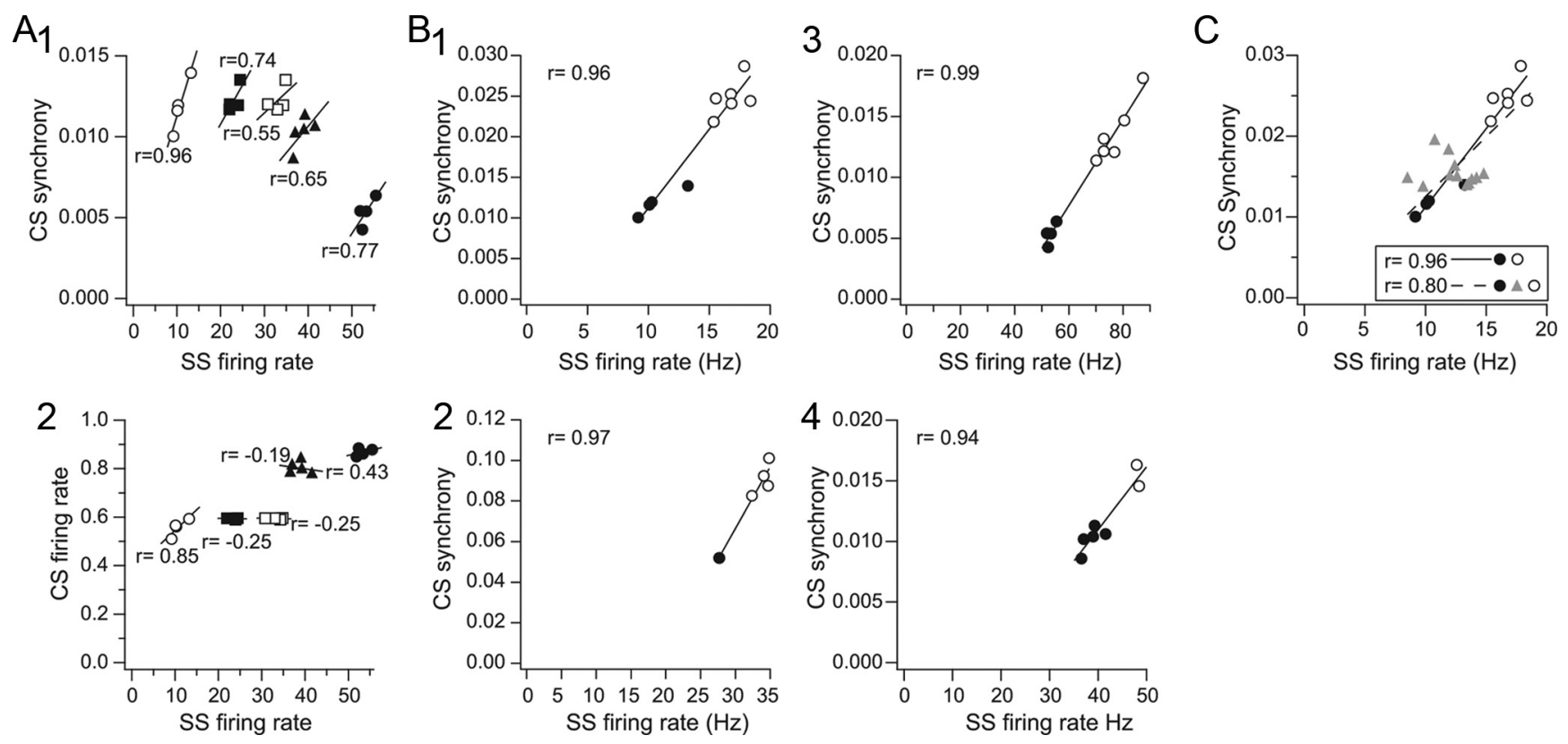

Figure 6. Correlation of CS activity and SS firing rates. $\boldsymbol{A}$, Scatter plots of SS firing rate against CS synchrony (A1) and CS firing rate (A2) under control conditions. Each set of symbols refers to data from one experiment. SS firing rates and CS synchrony were calculated for sequential 5 min periods during control periods of 20 min or longer. Regression lines are least-squares fits to the data, and the $r$ values are the correlation coefficients for each dataset. $\boldsymbol{B}$, Correlation of changes in CS synchrony and SS firing rates with picrotoxin. Scatter plots from four ( $\boldsymbol{B} \mathbf{1}-\boldsymbol{B} 4$ ) experiments are shown in which recordings were made in control (filled circles) and during application of picrotoxin by Gelfoam (unfilled circles). As in $\boldsymbol{A}$, each data point is from a 5 min recording period. The picrotoxin data shown are from periods $20-40$ min after the start of the drug application. Control periods for experiments 1, 3, and 4 are replotted from $\boldsymbol{A} 1$ (unfilled circles, filled circles, filled triangles, respectively). $\boldsymbol{C}$, Scatter plot showing intermediate picrotoxin periods along with control and final picrotoxin period from experiment 1 of $\boldsymbol{B}$.

level were calculated for each period. In each case, a positive correlation (range, 0.55-0.96) was observed between these variables (Fig. 6A1) ( $n=5$ cells with SSs, 98 cells with CSs, 4 animals). In contrast, in control, the CS firing rate was relatively weakly correlated with SS activity, but most often ( 3 of 5 SS cells) showed an inverse relationship (Fig. 6A2), as would be expected based on previous studies that have shown that CS and SS activity levels tend to be inversely related.

Although the association of CS synchrony and SS firing rate was consistently observed, the range of average SS firing rates displayed by any particular cell in control was relatively limited (i.e., varied by $<10 \mathrm{~Hz}$ ) (Fig. 6A1). During behavior much greater variations in SS activity occur, so to investigate the correlation of CS and SS activity over a larger range of SS activity we compared CS and SS activity in control and picrotoxin conditions. In every case picrotoxin resulted in an increase in both CS synchrony and SS activity ( $n=6$ SS cells, 115 CS cells, 5 animals). Four examples are shown in Figure $6 B$, in which the picrotoxin condition was divided into 5 min segments, like what was done for the control period. In these plots the control period data are indicated by filled circles and the picrotoxin data by open circles. The separation of the two sets of circles clearly shows the effect of picrotoxin to increase both SS firing rate and CS synchrony. Even over this wider range of SS firing rates, a strong relatively linear relationship holds, with the correlation coefficient, $r,>0.90$ in each case. One should note that the picrotoxin periods shown were taken after picrotoxin had shown effects throughout the array, some time (20-40 min) after the start of the drug application. Use of this later picrotoxin period ensured that the area in which SSs were being recorded had been affected by picrotoxin. However, use of this later period is why there is a gap between the control and picrotoxin data in the plots of Figure $6 \mathrm{~B}$. The data from earlier periods during picrotoxin application tended to yield data points that filled in the gap (Fig. $6 C$, triangles), as would be predicted; however, data from these periods showed more scatter and typically fell off the line created by the control and later picrotoxin data. These features are most likely due to picrotoxin affecting cells in part of the array before and after the area from which the SSs were recorded (e.g., if picrotoxin increased CS synchrony in part of the multielectrode array before it reached the part at which SS activity was also being recorded, this would result in average CS synchrony rising without a change in SS activity).

\section{Post-CS pause and modulation of SS activity are reduced by picrotoxin}

It is well known that there is a brief pause in SS activity following a CS. In addition, following this pause a short-term modulation of SS activity, either excitatory or inhibitory, can occur (McDevitt et al., 1982). These changes in SS activity have been ascribed to some combination of inactivation of the Purkinje cell membrane by the CS and activation of the Golgi, basket, or stellate cells by collaterals of olivocerebellar axons (Bloedel and Roberts, 1971; Latham and Paul, 1971; Burg and Rubia, 1972; Colin et al., 1980; Ekerot and Oscarsson, 1980, 1981; Rawson and Tilokskulchai, 1981). In our recordings $(n=6)$, CS-triggered SS histograms from control activity revealed cells with pauses followed by a transient decrease $(n=2)$ (Fig. $7 A 1)$ or increase in SS $(n=3)$ (Fig. 7B1) activity, or an immediate return to the pre-CS baseline level of activity $(n=1)$. In all cases $(n=6)$, during application of picrotoxin to the cortex, the postpause transient change in SS activity was altered (if originally present) and the pause itself appeared to be shortened (control, $14.6 \pm 4.9 \mathrm{~ms}$; picrotoxin, $7.6 \pm 4.0 \mathrm{~ms} ; p=0.069$ ) (Fig. 7). Although the difference in pause duration did not reach statistical significance, this was likely due in part to our inability to measure the full reduction in the pause because a dead time of -2 to $5 \mathrm{~ms}$ surrounding each CS was used during spike detection (to avoid counting the CS as a SS). In fact, 

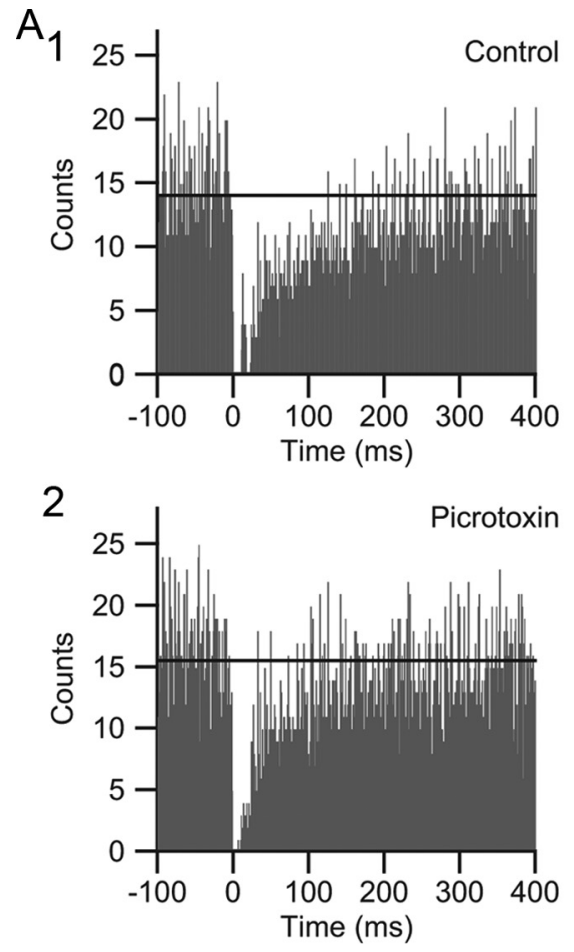
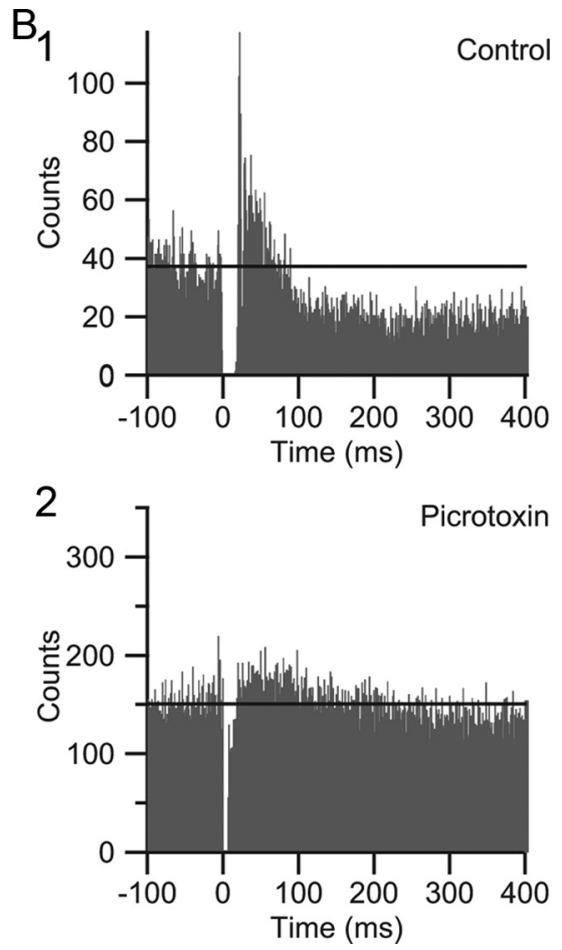

toxin (38/39 cells). In one cell, however, picrotoxin resulted in the presence of an extra spikelet. Thus, the number of axonic spikes associated with each CS is likely to have remained the same in the two conditions for nearly all Purkinje cells, making changes in CS waveform an unlikely mechanism for explaining the observed increase in CS synchrony with picrotoxin. Consistent with this conclusion, in two of the four experiments the large majority of the analyzed cells ( $70 \%$ and $71 \%)$ did not show changes in CS waveforms between conditions (and yet showed increased CS synchrony).

Last, to verify that the signals we recorded during picrotoxin application still reflected CS activity generated by the olivocerebellar system despite some changes in waveform, lidocaine was injected into the IO following the picrotoxin session in two experiments. Within minutes of the start of the injection, lidocaine completely eliminated the spike activity in the cerebellar cortex, confirming its identity as CSs generated as a result of olivocerebellar activity (Fig. $8 C$ ).

Figure 7. Post-CS pause and modulation of SS activity are altered by Gelfoam application of picrotoxin. $A, B$, CS-triggered SS histograms from two cells. In control, one cell (A1) shows a postpause reduction in SS activity that lasts several hundred milliseconds, and the other cell (B1) shows an initial increase and then reduction in SS activity following the pause. Application of picrotoxin to the cortex leads to a reduction of the post-CS modulation of SS activity in both cells (compare $\boldsymbol{A} 1$ vs $\boldsymbol{A} \mathbf{2}$ and $\boldsymbol{B} 1$ vs $\boldsymbol{B} \mathbf{2}$ ). Note also the shortening of the pause period, which is most easily seen for the cell in $\boldsymbol{B}$. To avoid counting CS spikelets as SSS, no activity was counted for -2 to $5 \mathrm{~ms}$ surrounding the $0 \mathrm{~ms}$ bin, and therefore the absence of counts in these bins is due to that procedure. The horizontal lines show the average bin height for the $100 \mathrm{~ms}$ before the CS. The cell in $A$ is the same cell as in Figure 5.

in three of six cells the pause was shortened to this $5 \mathrm{~ms}$ limit, which represented a $63 \%$ reduction. Overall, the results support the idea that GABAergic activity is a factor in shaping both the pause and postpause modulation of SS activity following a CS.

\section{Cortical application of picrotoxin can alter the CS waveform}

With application of picrotoxin to the cerebellar cortex, CSs retained their basic identity; however, small alterations of the waveform occurred in some cases. Such changes are not entirely surprising, as activation of cortical inhibitory interneurons can affect the morphology of the CS (Eccles et al., 1966b), and they raise the possibility that the effect of cortical picrotoxin could be mediated, at least in part, by changes in the CS itself, because the CS waveform in the dendrites and somata of the Purkinje cell have been shown to be related to the number of the axonic spikes triggered by the CS (Mathy et al., 2009). To investigate this issue the CS waveforms were analyzed from four experiments in which application of picrotoxin increased CS synchrony. In each experiment, only those cells $(n=39)$ whose CSs in the control condition comprised bursts containing relatively large and stereotyped spikelets were analyzed in detail. For these cells, between 50 and 200 CSs were averaged for each cell in the control and picrotoxin conditions. In all cases, the initial spike of the burst was essentially identical in the control and picrotoxin conditions. Many cells $(16 / 39)$ also showed virtually no change in the spikelets of the burst (Fig. 8A). A small majority (22/39), however, did show a consistent increase in the intraburst interspike interval in the range of 100-500 $\mu \mathrm{s}$ (Fig. 8 B). For almost all cells the number of spikes within a burst did not change between control and picro-

\section{Discussion}

The central finding of this study is that changes in SS activity levels significantly alter CS firing rates and synchrony. Moreover, the effects of SS changes are spatially restricted to the same narrow rostrocaudally running strip of cortex in which they take place. The results also provide further evidence that the post-CS pause and modulation of SS activity are mediated, at least in part, by cortical GABAergic activity, and that such activity can also influence the CS waveform. In sum, the results suggest that each cerebellar cortical region exerts control over its own CS synchrony patterns and thus that the connections between the cerebellum and IO form a series of closed loops such that activity in each structure helps organize the impending activity in the other.

\section{Cerebellar control of CS synchrony}

Although CS synchrony is often described as having a rostrocaudal banding pattern (Sasaki et al., 1989; Sugihara et al., 1993; Lang et al., 1999), this description refers to its average distribution. Normally, a complete "band" of Purkinje cells firing CSs simultaneously is a relatively rare occurrence. Rather, CSs of any particular cell are synchronized with those of varying subsets of cells, and the banding pattern arises because the cells most often included in these subsets belong to the same rostrocaudally running cortical strip as the cell itself. Thus, CS synchrony patterns are dynamic, and are not strictly determined by anatomical factors, such as the topography of the olivocerebellar projection, although such factors do play a role (Sugihara et al., 2007).

Electrical coupling of IO neurons is required for CS synchrony (Llinas et al., 1974; Sotelo et al., 1974; Leznik and Llinás, 2005; Blenkinsop and Lang, 2006; Marshall et al., 2007); however, the specific synchrony patterns are determined mainly by the activity of GABAergic and glutamatergic IO afferents (Lang et al., 1996; 
Lang, 2001, 2002). These afferents project both to the somata and dendrites of IO neurons, and to dendritic spines within the IO glomeruli (de Zeeuw et al., 1989a,b; Nelson and Mugnaini, 1989; Fredette and Mugnaini, 1991), and thus likely control both the general excitability of IO neurons and the effective coupling between specific pairs of IO neurons (Lang, 2001, 2002). The GABAergic afferents to most IO regions arise from the cerebellar nuclei, and the cerebellar nuclei, in turn, receive most $(\sim 75 \%)$ of their synaptic input from Purkinje cells (Palkovits et al., 1977; De Zeeuw and Berrebi, 1995). Thus, the cerebellar cortex, via its influence on the cerebellar nuclei, should help determine the CS synchrony patterns. Our results provide evidence that this idea is correct: application of picrotoxin and muscimol to the cortex led to significant increases and decreases, respectively, in CS synchrony.

Although CSs and SSs together form the efferent activity of the cerebellar cortex, the direct action of picrotoxin and muscimol that led to changes in CS synchrony almost certainly had to be to alter SS activity, because CSs are triggered by the firing of IO neurons, and IO cells would not be directly affected by drugs applied to the cerebellar cortex. Our results support this conclusion. Spontaneous changes in SS rates were correlated with changes in CS synchrony. Local application of picrotoxin to the cerebellar cortex increased SS firing rates (present results and Thomsen et al., 2004), and the magnitude of these increases was correlated with CS synchrony changes. Conversely, muscimol silences SS activity (Vigot et al., 1993), and we found that it decreased CS synchrony as well.

Given that both neurons in the cortico-nucleo-IO pathway are inhibitory (Ito et al., 1964, 1970; Nelson and Mugnaini, 1989), the correlation between SS firing rate and CS synchrony probably results from the following sequences of events: increased SS activity inhibits the cerebellar nuclei, leading to reduced release of GABA in the IO, which causes an increase in CS synchrony; and decreased SS activity disinhibits the cerebellar nuclei, leading to increased intra-IO GABA release, and thereby decreased CS synchrony.

There is evidence for the above sequences. Tonically increased SS activity following IO lesions causes a decrease in cerebellar nuclear firing (Batini and Billard, 1985), and picrotoxin applied to the cerebellar cortex lowers cerebellar nuclear firing rates (Blenkinsop and Lang, unpublished observations). Moreover, our previous finding that block of GABA within the IO or lesion of the nuclei causes increased CS synchrony (Lang et al., 1996; Lang, 2002) suggests that increased nuclear activity would decrease CS synchrony.

In sum, the evidence suggests that SS changes are a major mechanism for regulating CS synchrony. However, although changes in CS firing rates and synchrony could not have been the initiating cause of those changes, CSs trigger axonal spikes (Ito and Simpson, 1971; Campbell and Hesslow, 1986; Khaliq and Raman, 2005; Monsivais et al., 2005), and therefore could potentially affect nuclear activity, and thereby the IO. Thus, an initial SS-induced change in CS activity could lead to feedback to the IO that further alters CS activity.

Changes in CS firing rates theoretically could change the amount of inhibition of the cerebellar nuclei; however, CS rates
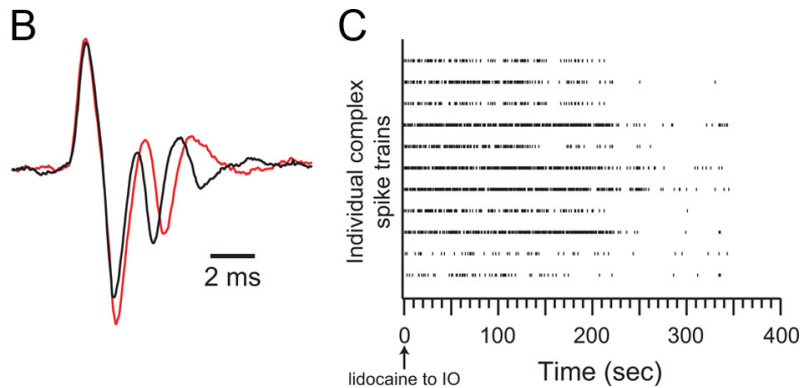

toxin. $A, B$, Averaged extracellular records of $C S$ activity before (black traces) and during (red traces) picrotoxin application to the cerebellar cortex. The cell in $\boldsymbol{A}$ showed no change in $C S$ waveform, whereas the cell in $\boldsymbol{B}$ showed a lengthening of the intra-CS interspike interval. Time calibration in $\boldsymbol{B}$ applies to both $\boldsymbol{A}$ Intraolivary lidocaine injections eliminate CS activity. Raster display of experiment in which lidocaine was injected into the presents the CSs from one Purkinje cell. In this experiment 11 cells were recorded simultaneously. CS activity disappeared 6-7

are so low compared with SS rates, that changes in them would alter overall Purkinje cell spike output by only a few percent in most situations. Thus, changes in CS firing rates probably make little direct contribution to the final level of CS synchrony. Indeed, CS and SS firing rates are inversely related in many cases (de Montigny and Lamarre, 1973; Colin et al., 1980; Rawson and Tilokskulchai, 1981; Demer et al., 1985), and in the situation in which CS firing rates are raised and SSs activity is decreased, the net result is an increase in nuclear firing rate (Armstrong et al., 1979; Andersson and Hesslow, 1987), suggesting that changes in SS firing rates dominate those in CS firing rates.

Even if changes in CS firing rates are not a major determinant of CS synchrony levels, CS synchrony itself still may be one, because synchronous CS discharges can transiently silence cerebellar nuclear neurons for hundreds of milliseconds (Blenkinsop and Lang, unpublished data), and thus presumably shut off GABA release in the IO for significant periods of time. Synchronous CSs can have such a strong effect on nuclear activity because of the large convergence of Purkinje axons onto each nuclear neuron (Palkovits et al., 1977). Whether this effect or the effect of changes in SS activity is stronger is difficult to say. However, when CS synchrony and firing rates are enhanced by intra-IO picrotoxin injections (Lang et al., 1996; Lang, 2002), an overall increase in cerebellar nuclear firing occurs (Blenkinsop and Lang, 2005), which suggests the concomitant decrease in SS activity is the dominant factor. Thus, while increased CS synchrony likely contributes to its own generation, changes in SS activity are probably more important.

Before concluding that cerebellar cortical output, and in particular SSs, exert dynamic control over the synchronous CS activity, we consider whether the results can be explained by the drugs acting at noncortical sites. The closest structures to the cerebellar cortex are the cerebellar nuclei, and therefore they are the most likely to have been affected if the drugs had diffused beyond the cortex. Given that GABAergic terminals form $\sim 75 \%$ of all presynaptic boutons in the nuclei (Palkovits et al., 1977; De Zeeuw and Berrebi, 1995), nuclear firing rates should have increased if significant concentrations of picrotoxin, for example, had occurred there. If this had happened, however, CS firing rates would have decreased (contrary to what was observed), as stimulation of nucleo-olivary fibers inhibits CS activity (Bengtsson et al., 2004). Nuclear cell firing, in fact, decreases with cortical application of picrotoxin (Blenkinsop and Lang, unpublished results). Conversely, if muscimol had diffused to the nuclei and 
silenced them, CS firing rates should have increased (again contrary to what was observed), as lesions of the nuclei increase CS activity (Lang et al., 1996).

Furthermore, the differential magnitude and time course of the synchrony changes in the two halves of the recording array when drugs were applied lateral to the array (and the absence of these differentials when picrotoxin was applied rostrally) strongly indicate that the drugs were acting locally on the cerebellar cortex, rather than at the cerebellar nuclei or other more distant brain regions. For example, had picrotoxin reached the IO, it is unlikely that the increase in synchrony would have always occurred first on the lateral half of the array when picrotoxin was applied lateral to the array. Most likely CS synchrony would have increased uniformly across the array, or the part of the recording array first experiencing an increase in synchrony would have varied randomly. Even if some part of the IO does receive, or respond to, picrotoxin preferentially, variation in first response region would have occurred, because the exact placement of the recording array varied between experiments, so that in each experiment the array covered somewhat different regions of crus $2 \mathrm{a}$, which receive input from distinct regions of the IO (Sugihara and Shinoda, 2004).

\section{Local cortical control of CS synchrony}

A major implication of the present results is that control of CS synchrony is a localized function of the cortex, and, more specifically, that the SS activity of each region of crus $2 \mathrm{a}$ acts to regulate the level of CS synchrony in that region. Indeed, cortical control of CS synchrony may be localized to regions $<1 \mathrm{~mm}$ in width along the longitudinal folial axis. This result is consistent with the precise topography and alignment of the projections between the cerebellum and IO that have been demonstrated anatomically (Groenewegen and Voogd, 1977; Groenewegen et al., 1979; Voogd and Bigaré, 1980; Van der Want et al., 1989; BuisseretDelmas and Angaut, 1993). It is also consistent with the correspondence of CS synchrony bands and zebrin compartments (Sugihara et al., 2007), which are typically 250-500 $\mu \mathrm{m}$ wide for lateral crus $2 \mathrm{a}$, and that these compartments receive climbing fibers arising from highly restricted IO regions (Sugihara and Shinoda, 2004), and project to relatively restricted regions within the cerebellar nuclei (Sugihara and Shinoda, 2007).

In sum, the picture that emerges is one in which each cortical area exerts control over its own CS synchrony. In particular, the inverse modulation of SS and CS firing likely maintains the tonic levels of nuclear activity and acts as a negative feedback to prevent excessive levels of CS synchrony, which lead to abnormal motor function, including tremor.

\section{References}

Andersson G, Hesslow G (1987) Activity of Purkinje cells and interpositus neurones during and after periods of high frequency climbing fibre activation in the cat. Exp Brain Res 67:532-542.

Armstrong DM, Cogdell B, Harvey RJ (1979) Discharge patterns of Purkinje cells in cats anaesthetized with alpha-chloralose. J Physiol 291:351-366.

Batini C, Billard JM (1985) Release of cerebellar inhibition by climbing fiber deafferentation. Exp Brain Res 57:370-380.

Bengtsson F, Svensson P, Hesslow G (2004) Feedback control of Purkinje cell activity by the cerebello-olivary pathway. Eur J Neurosci 20:2999-3005.

Blenkinsop TA, Lang EJ (2005) Synchronous complex spike activity is associated with changes in deep cerebellar nuclear firing patterns. Soc Neurosci Abstr 31:179.1

Blenkinsop TA, Lang EJ (2006) Block of inferior olive gap junctional coupling decreases Purkinje cell complex spike synchrony and rhythmicity. J Neurosci 26:1739-1748.
Bloedel JR, Roberts WJ (1971) Action of climbing fibers in cerebellar cortex of the cat. J Neurophysiol 34:17-31.

Buisseret-Delmas C, Angaut P (1993) The cerebellar olivo-corticonuclear connections in the rat. Prog Neurobiol 40:63-87.

Burg D, Rubia FJ (1972) Inhibition of cerebellar Purkinje cells by climbing fiber input. Pflugers Arch 337:367-372.

Campbell NC, Hesslow G (1986) The secondary spikes of climbing fibre responses recorded from Purkinje cell axons in cat cerebellum. J Physiol 377:225-235.

Colin F, Manil J, Desclin JC (1980) The olivocerebellar system. I. Delayed and slow inhibitory effects: an overlooked salient feature of cerebellar climbing fibers. Brain Res 187:3-27.

de Montigny C, Lamarre Y (1973) Rhythmic activity induced by harmaline in the olivo-cerebello-bulbar system of the cat. Brain Res 53:81-95.

De Zeeuw CI, Berrebi AS (1995) Postsynaptic targets of Purkinje cell terminals in the cerebellar and vestibular nuclei of the rat. Eur J Neurosci 7:2322-2333.

De Zeeuw CI, Ruigrok TJ (1994) Olivary projecting neurons in the nucleus of Darkschewitsch in the cat receive excitatory monosynaptic input from the cerebellar nuclei. Brain Res 653:345-350.

de Zeeuw CI, Holstege JC, Ruigrok TJ, Voogd J (1989a) Ultrastructural study of the GABAergic, cerebellar, and mesodiencephalic innervation of the cat medial accessory olive: anterograde tracing combined with immunocytochemistry. J Comp Neurol 284:12-35.

de Zeeuw CI, Holstege JC, Ruigrok TJH, Voogd J (1989b) The cerebellar, mesodiencephalic and GABAergic innervation of the glomeruli in the cat inferior olive. A comparison at the ultrastructural level. In: The olivocerebellar system in motor control (Strata P, ed), pp 111-116. Berlin: Springer.

de Zeeuw CI, Ruigrok TJ, Holstege JC, Jansen HG, Voogd J (1990) Intracellular labeling of neurons in the medial accessory olive of the cat: II. Ultrastructure dendritic spines and the GABAergic innervation. J Comp Neurol 300:478-494.

Demer JL, Echelman DA, Robinson DA (1985) Effects of electrical stimulation and reversible lesions of the olivocerebellar pathway on Purkinje cell activity in the flocculus of the cat. Brain Res 346:22-31.

Eccles JC, Llinás R, Sasaki K (1964) Excitation of cerebellar Purkinje cells by the climbing fibres. Nature 203:245-246.

Eccles JC, Llinás R, Sasaki K (1966a) The excitatory synaptic action of climbing fibers on the Purkinje cells of the cerebellum. J Physiol 182:268-296.

Eccles JC, Llinás R, Sasaki K, Voorhoeve PE (1966b) Interaction experiments on the responses evoked in Purkinje cells by climbing fibres. J Physiol 182:297-315.

Ekerot CF, Oscarsson O (1980) Prolonged dendritic depolarizations evoked in Purkinje cells by climbing fibre impulses. Brain Res 192:272-275.

Ekerot CF, Oscarsson O (1981) Prolonged depolarization elicited in Purkinje cell dendrites by climbing fibre impulses in the cat. J Physiol 318:207-221.

Fredette BJ, Mugnaini E (1991) The GABAergic cerebello-olivary projection in the rat. Anat Embryol 184:225-243.

Gerstein GL, Kiang NY (1960) An approach to the quantitative analysis of electrophysiological data from single neurons. Biophys J 1:15-28.

Granit R, Phillips CG (1956) Excitatory and inhibitory processes acting upon individual Purkinje cells of the cerebellum in cats. J Physiol 133:520-547.

Groenewegen HJ, Voogd J (1977) The parasagittal zonation within the olivocerebellar projection. I. Climbing fiber distribution in the vermis of cat cerebellum. J Comp Neurol 174:417-488.

Groenewegen HJ, Voogd J, Freedman SL (1979) The parasagittal zonation within the olivocerebellar projection. II. climbing fiber distribution in the intermediate and hemispheric parts of the cat cerebellum. J Comp Neurol 183:551-601.

Horn KM, Hamm TM, Gibson AR (1998) Red nucleus stimulation inhibits the inferior olive. J Neurophysiol 80:3127-3136.

Ito M (1989) Long-term depression. Annu Rev Neurosci 12:85-102.

Ito M (2002) Historical review of the significance of the cerebellum and the role of Purkinje cells in motor learning. Ann N Y Acad Sci 978:273-288.

Ito M, Simpson JI (1971) Discharges in Purkinje cell axons during climbing fiber activation. Brain Res 31:215-219.

Ito M, Yoshida M, Obata K (1964) Monosynaptic inhibition of the intracerebellar nuclei induced from the cerebellar cortex. Experientia (Basel) 20:575-576. 
Ito M, Yoshida M, Obata K, Kawai N, Udo M (1970) Inhibitory control of intracerebellar nuclei by the Purkinje cell axons. Exp Brain Res 10:64-80.

Jahnsen H (1986a) Electrophysiological characteristics of neurones in the guinea-pig deep cerebellar nuclei in vitro. J Physiol 372:129-147.

Jahnsen H (1986b) Extracellular activation and membrane conductances of neurones in the guinea-pig deep cerebellar nuclei in vitro. J Physiol 372:149-168.

Khaliq ZM, Raman IM (2005) Axonal propagation of simple and complex spikes in cerebellar Purkinje neurons. J Neurosci 25:454-463.

King JS (1976) The synaptic cluster (glomerulus) in the inferior olivary nucleus. J Comp Neurol 165:387-400.

Lang EJ (2001) Organization of olivocerebellar activity in the absence of excitatory glutamatergic input. J Neurosci 21:1663-1675.

Lang EJ (2002) GABAergic and glutamatergic modulation of spontaneous and motor-cortex-evoked complex spike activity. J Neurophysiol 87:1993-2008.

Lang EJ, Sugihara I, Llinás R (1996) GABAergic modulation of complex spike activity by the cerebellar nucleoolivary pathway in rat. J Neurophysiol 76:255-275.

Lang EJ, Sugihara I, Welsh JP, Llinás R (1999) Patterns of spontaneous Purkinje cell complex spike activity in the awake rat. J Neurosci 19:27282739.

Latham A, Paul DH (1971) Spontaneous activity of cerebellar Purkinje cells and their responses to impulses in climbing fibres. J Physiol 213:135-156.

Leznik E, Llinás R (2005) Role of gap junctions in synchronized neuronal oscillations in the inferior olive. J Neurophysiol 94:2447-2456.

Llinas R, Baker R, Sotelo C (1974) Electrotonic coupling between neurons in cat inferior olive. J Neurophysiol 37:560-571.

Llinás R, Mühlethaler M (1988) Electrophysiology of guinea-pig cerebellar nuclear cells in the in vitro brain stem-cerebellar preparation. J Physiol 404:241-258

Marshall SP, Van der Giessen RS, De Zeeuw CI, Lang EJ (2007) Altered olivocerebellar activity patterns in the connexin 36 knockout mouse. Cerebellum 6:287-299.

Mathy A, Ho SS, Davie JT, Duguid IC, Clark BA, Häusser M (2009) Encoding of oscillations by axonal bursts in inferior olive neurons. Neuron 62:388-399.

McDevitt CJ, Ebner TJ, Bloedel JR (1982) The changes in Purkinje cell simple spike activity following spontaneous climbing fiber inputs. Brain Res 237:484-491.

Monsivais P, Clark BA, Roth A, Häusser M (2005) Determinants of action potential propagation in cerebellar Purkinje cell axons. J Neurosci 25:464-472.

Montarolo PG, Palestini M, Strata P (1982) The inhibitory effect of the olivocerebellar input on the cerebellar Purkinje cells in the rat. J Physiol 332:187-202.

Nelson BJ, Mugnaini E (1989) Origins of GABAergic inputs to the inferior olive. In: The olivocerebellar system in motor control (Strata P, ed). Berlin: Springer.

Palkovits M, Mezey E, Hámori J, Szentágothai J (1977) Quantitative histo- logical analysis of the cerebellar nuclei in the cat. I. Numerical data on cells and on synapses. Exp Brain Res 28:189-209.

Rawson JA, Tilokskulchai K (1981) Suppression of simple spike discharge of cerebellar Purkinje cells by impulses in climbing fibre afferents. Neurosci Lett 25:125-130.

Sasaki K, Bower JM, Llinás R (1989) Multiple Purkinje cell recording in rodent cerebellar cortex. Eur J Neurosci 1:572-586.

Sato Y, Miura A, Fushiki H, Kawasaki T (1992) Short-term modulation of cerebellar Purkinje cell activity after spontaneous climbing fiber input. J Neurophysiol 68:2051-2062.

Savio T, Tempia F (1985) On the Purkinje cell activity increase induced by suppression of inferior olive activity. Exp Brain Res 57:456-463.

Schwarz C, Welsh JP (2001) Dynamic modulation of mossy fiber system throughput by inferior olive synchrony: a multielectrode study of cerebellar cortex activated by motor cortex. J Neurophysiol 86:2489-2504.

Simpson JI, Wylie DR, De Zeeuw CI (1996) On climbing fiber signals and their consequence(s). Behav Brain Sci 19:384-398.

Sotelo C, Llinas R, Baker R (1974) Structural study of inferior olivary nucleus of the cat: morphological correlates of electrotonic coupling. J Neurophysiol 37:541-559.

Sugihara I, Shinoda Y (2004) Molecular, topographic, and functional organization of the cerebellar cortex: a study with combined aldolase $\mathrm{C}$ and olivocerebellar tracing. J Neurosci 24:8771-8785.

Sugihara I, Shinoda Y (2007) Molecular, topographic, and functional organization of the cerebellar nuclei: analysis by three-dimensional mapping of the olivonuclear projection and aldolase C labeling. J Neurosci 27:9696-9710.

Sugihara I, Lang EJ, Llinás R (1993) Uniform olivocerebellar conduction time underlies Purkinje cell complex spike synchronicity in the rat cerebellum. J Physiol 470:243-271.

Sugihara I, Marshall SP, Lang EJ (2007) Relationship of complex spike synchrony to the lobular and longitudinal aldolase $\mathrm{C}$ compartments in crus IIA of the cerebellar cortex. J Comp Neurol 501:13-29.

Thomsen K, Offenhauser N, Lauritzen M (2004) Principal neuron spiking: neither necessary nor sufficient for cerebral blood flow in rat cerebellum. J Physiol 560:181-189.

Van der Want JJ, Wiklund L, Guegan M, Ruigrok T, Voogd J (1989) Anterograde tracing of the rat olivocerebellar system with Phaseolus vulgaris leucoagglutinin (PHA-L). Demonstration of climbing fiber collateral innervation of the cerebellar nuclei. J Comp Neurol 288:1-18.

Vigot R, Billard JM, Batini C (1993) Reduction of GABA inhibition in Purkinje and cerebellar nuclei neurons in climbing fibre deafferented cerebella of rat. Neurosci Res 17:249-255.

Voogd J, Bigaré F (1980) Topographical distribution of olivary and corticonuclear fibers in the cerebellum. A review. In: The inferior olivary nucleus: anatomy and physiology (Courville J, de Montigny C, Lamarre Y, eds), pp 207-234. New York: Raven.

Weiss C, Houk JC, Gibson AR (1990) Inhibition of sensory responses on cat inferior olive neurons produced by stimulation of red nucleus. J Neurophysiol 64:1170-1185. 